\title{
LXVIII. The action of the radium emanation upon water
}

\section{Prof. E. Rutherford F.R.S. \& T. Royds M.Sc.}

To cite this article: Prof. E. Rutherford F.R.S. \& T. Royds M.Sc. (1908) LXVIII. The action of the radium emanation upon water, Philosophical Magazine Series 6, 16:95, 812-818, DOI: 10.1080/14786441108636558

To link to this article: http://dx.doi.org/10.1080/14786441108636558

$$
\text { 曲 Published online: } 21 \text { Apr } 2009 .
$$

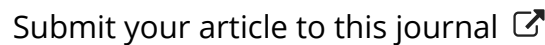

Џ Article views: 4

Q View related articles $₫$ 


\section{[ 812$]$}

LXVIII. The Action of the Radium Enanation upon Water. $B y$ Prof. E. Rutherford, F.R.S., and T. Royds, M.Sc., 1851 Exhibition Science Scholar*.

YINCE the initial experiments of Ramsay and Soddy in - 1903, the production of helium from radium and its emanation have been completely substantiated by a number of independent observers. On the view that the a particle is a helium atom, the appearance of helium from radioactive matter in general receives a simple and satisfactory explanation.

Recently Mr. Cameron and Sir William Ramsay have attacked the important question as to whether the radiations from a large quantity of radium emanation are effective in transforming the atoms of ordinary matter. They have published results to prove that under the influence of the radium emanation, copper is transformed into lithium and possibly into sodium and potassium. In addition they have given evidence that in the presence of copper solutions the emanation disintegrates into argon, and in the presence of water into neon. A complete and satisfactory proof of these transformations is attended by great experimental difficulty. In a recent paper $\dagger$, Cameron and Ramsay sum up the results of their experiments as follows:- "In carrying out such work it is extremely difficult to prevent traces of air leaking into the apparatus during the considerable length of time which must elapse before an experiment is completed. Since $0 \cdot 1$ c.c. of atmospheric nitrogen contains sufficient argon to be detected spectroscopically after the nitrogen has been removed by sparking, the proof that the presence of argon is due to some other cause is rendered extremely difficult. Similarly, it is far from easy to free copper solutions completely trom traces of lithium, and to prove convincingly that lithium did not pre-exist in such solution treated with emanation. The detection of neon is open to no such objection. The only possible source, other than transmutation, is the aluminium of the electrodes. The vacuum-tube had been previously run a great number of times at different pressures, washed out with air, and finally showed traces of hydrogen, and hydrogen alone. It is inconceivable that neon can bave resulted thus by chance from two experiments with water, where in each case the residues were tested between those from numerous similar experiments with other solutions in which no neon was detected. We must regard

* Communicated by the Authors.

† Journ. Chem. Soc. June 1908, p. 992. 
the transformation of emanation into neon, in presence of water, as indisputably proved, and, if a transformation be defined as a transformation brought about at will, by change of conditions, then this is the first case of transmutation of which conclusive evidence is put forward."

Mme. Curie and Mlle. Gleditsch* have recently repeated the experiments of Cameron and Ramsay with reference to the transformation of copper into lithium, and have obtained no certain evidence of the production of lithium.

With the aid of the radium loaned by the Vienna Academy of Sciences, we have made experiments to see whether neon is produced when the radium emanation disintegrates in the presence of water. Thanks to the use of the absorbing properties of charcoal, the detection of a minute quantity of neon is now a comparatively simple matter. Coconut charcoal at the temperature of liquid air absorbs all gases except neon and helium. The spectroscopic test of the presence of neon is unmistakable on account of its very characteristic spectrum of a bright line in the yellow and a group of bright lines in the red. Since there could be no possible doubt that neon was present in the experiments of Cameron and Ramsay, the question arose whether the neon observed could have been derived from the air; for Strutt $\dagger$ has recently shown that the presence of neon can be spectroscopically detected in ${ }_{10}^{3}$ c.c. of atmospheric air. Cameron and Ramsay state that in their experiments it was impossible to avoid a small leakage of air into their apparatus during the week or more that an experiment was in progress.

Before beginning the main investigation, a number of experiments were made to determine the amount of neon that could be detected spectroscopically. The apparatus, already described for the purification of the emanation $\ddagger$, was used for this purpose. The arrangement will be clearly seen from fig. 1.

The whole apparatus was first completely exhausted by means of a mercury-pump. A known volume of air contained in a small burette over mercury was introduced into the reservoir $\mathrm{R}$ by means of the mercury trough. The stopcocks $\mathrm{A}$ and $\mathrm{B}$ were closed and $\mathrm{C}$ opened, and the mercury raised to the level $\mathrm{D}$ by raising the reservoir $\mathrm{S}$. The stopcock $\mathrm{A}$ was opened to allow the gas to expand into the reservoir $\mathbf{E}$ of capacity about 30 c.c. and then closed. To the top of $\mathbf{E}$ was attached a small spectrum-tube $F$, of length about $7 \mathrm{cms}$.,

* Acad. Sciences, Aug. 10, 1908.

+ Proc. Roy. Soc. A. Ixxx. p. 572 (1908).

† Futherford, Phil. Mag. Aug. 1908. 


\section{Prof. E. Rutherford and Mr. T. Royds on the}

and of diameter $1 \mathrm{~mm}$., provided with thin platinum electrodes. A tube $\mathrm{H}$ containing a small quantity of coconut charcoal was attached to the side tube through a stopcock $\mathrm{L}$. Before the experiment the charcoal tube was thoroughly heated and

Fig. 1.

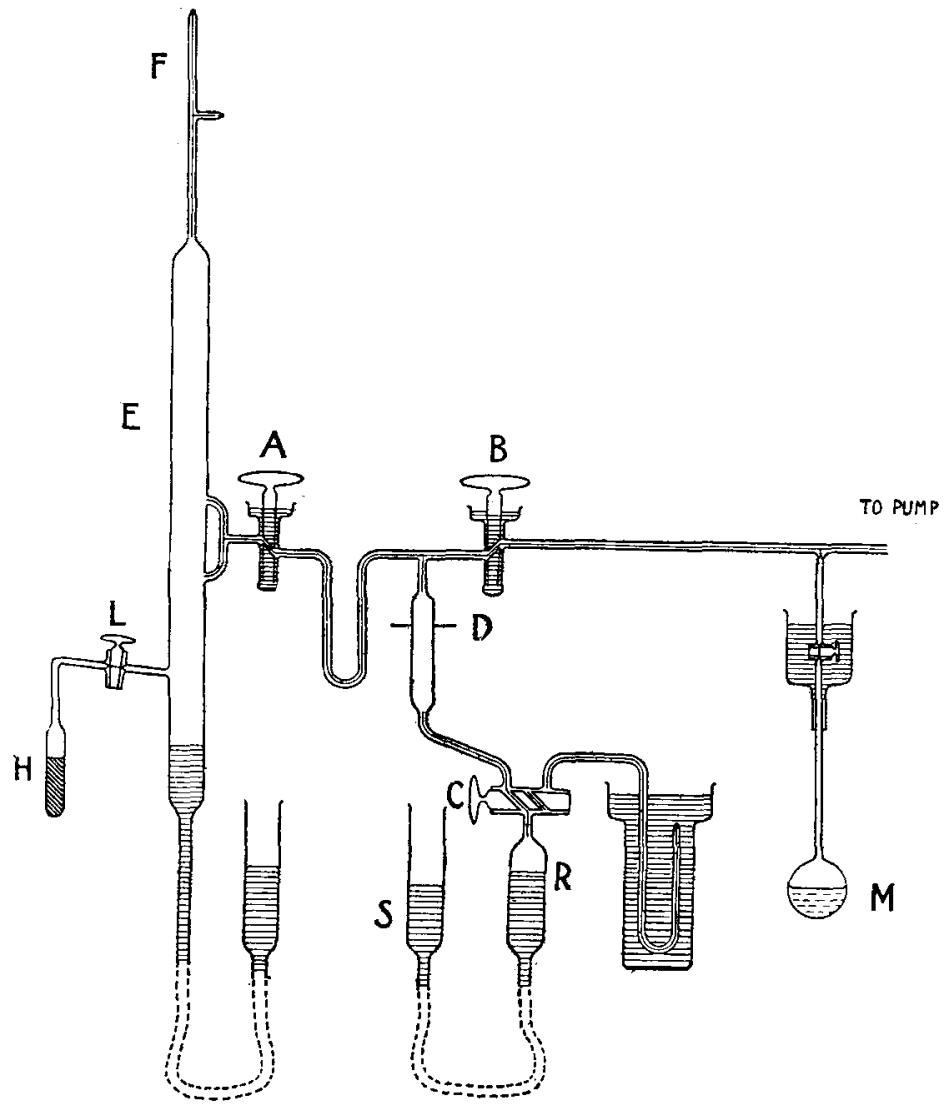

exhausted. On surrounding the tube $\mathrm{H}$ by liquid air, all the gases present in the air except neon and helium were absorbed. The stopcock L was then closed, and by raising the mercury reservoir the gases remaining in $\mathrm{E}$ were compressed into the vacuum-tube. On passing a discharge, the hydrogen and mercury lines were usually prominent, the former probably due to a trace of water vapour present. A pad of cottonwool was then wrapped round the spectrum-tube just above the level of the mercury and was soaked with liquid air. 
The spectrum of hydrogen and mercury disappeared, and the tube became very hard and showed only the neon spectrum.

Proceeding in this way, we were able to detect easily the neon yellow line when ${ }_{1}^{\frac{1}{5}}$ c.c. was introduced into the reservoir E. The conditions to bring out the spectrum most clearly could readily be tested by passing a discharge at short intervals through the tube as it slowly warmed up from evaporation of the liquid air. At a certain stage, the neon yellow line and the stronger reds flashed out clearly. With $\frac{2}{15}$ c.c. of air the red lines of neon were clearly visible, and with $\frac{1}{5}$ c.c. a brilliant spectrum of neon was obtained, showing most of the lines. These experiments were repeated on several occasions, the vacuum-tube being changed between each experiment, and in every case the same results were obtained.

According to the measurements of Ramsay neon is present in air in about one part in 100,000 by volume. The experiments thus show that one can readily detect the presence of neon in amount less than one millionth of a cubic centimetre. It is quite probable that with a still better choice of conditions, a still smaller quantity could be spectroscopically detected.

A Watts binocular grating spectroscope was found very convenient for a rapid examination of the spectra; measurements of wave-lengths were carried out by means of a Hilger direct-reading spectroscope.

\section{Experiments with the Emanation.}

In order to collect the gases formed by the action of radium emanation on water, we employed a method very similar to that used by Cameron and Ramsay. For clearness, we shall describe with some detail the progress of a complete experiment. A glass bulb of about 4 c.c. capacity, provided with a stopcock, was half filled with distilled water and then placed in an evaporation-bath to expel the air from the bulb and the water. The stopcock was then closed, and the bulb sealed on to a side-tube connected with the mercury-pump, as shown at $M$. The water in the bulb was frozen by surrounding it with liquid air and the last trace of air then pumped out. In the meantime the emanation, corresponding to the equilibrium amount from $150 \mathrm{mg}$. of radium, was carefully purified after the manner described in a previous paper and stored in the U-tube. By opening the stopcock $B$ the emanation was then all condensed in the frozen bulb. The stopcock was thereupon closed and surrounded by a 


\section{Prof. E. Rutherford and Mr. T. Royds on the}

mercury seal, to prevent any possible leakage of air into the bulb. At the end of three days, when it.was judged that the pressure of bydrogen and oxygen formed by the emanation from the water was about an atmosphere, the bulb was again frozen in liquid air and the non-condensable gases pumped out and collected. About 4 c.c. of mixed gases* were obtained, consisting mainly of hydrogen and oxygen. The gases were then introduced into a separate burette and exploded. A small quantity of pure electrolytic oxygen was then adder, and the gases again exploded to remove any excess of bydrogen. The residual gases (about $0-2$ c.c.) were not further treated, but passed directly into the receiver $\mathrm{E}$ in the manner already described, and the gases not absorbed by the charcoal were compressed into the vacuum-tube and spectroscopically examined. A complete and brilliant spectrum of helium was observed, but not a trace of the neon spectrum.

The greater part of the emanation had remained condensed in the bulb when the mixed gases were pumped out. The stopcock was closed and the seal again placed in position and the liquid air removed. The bulb was allowed to stand for six days, when about 4 c.c. of the mixed gases were again pumped out. As it might be possible that any neon formed would be dissolved in the water, the bulb was allowed to warm up to atmospheric temperature and then connected with the pump. The rapid evaporation of the water then gave a chance for removal of any absorbed gases into the pump. The small quantity of gas thus obtained was pumped out and added to the first quantity. The mixed gases were treated as before. A complete spectrum of helium was obtained but again no sign of the neon lines.

It might be thought that the presence of the helium in the discharge-tube would interfere with the detection of a small quantity of neon. A special experiment was made to test this point. At the end of the last experiment, the mercury was lowered below the level of the carbon tube and $\frac{1}{10}$ c.c. of air passed into the reservoir. After absorption by the charconl, the residual gases were tested spectroscopically as before. The neon spectrum was now clearly seen in addition to that of helium. When the lower part of the vacuum-tube was cooled to the temperature of liquid air by the pad of

* This amount of mixed gases is one third of that experimented with by Cameron and Ramsay, but the amount of neon to be expected from their results could have easily been detected with the smaller quantity. In experiments recorded later in the paper 30 c.c. of mixed gases were, used. 
cotton-wool, the yellow line of helium was distinctly brighter than the yellow line of neon, but at a certain stage of the warming up, the neon and helium spectra were about equal in brilliancy.

In all five experiments were made by this method, but in only one case was the presence of neon observed. This "as in the first experiment of the series. In order to see whether the presence of neon in this experiment could be ascribed to a leak of air into the apparatus, the charcoal was warmed up and the gases again pumped out and collected in a burette. The oxygen present was removed by melted phosphorus. About $1_{1}^{1}$ c.c. of gas remained, consisting mainly of nitrogen. Now $\frac{1}{15}$ c.c. of nitrogen corresponds to a leakage of about Io c.c. of air. The brightness of the neon yellow line compared with that of helium in the experiment was about that to be expected if this quantity of air had been added. This was confirmed by the observation that when an additional $\frac{1}{10}$ c.c. of air was added, the neon spectrum was increased notably in brightness.

\section{Experiments with the Gases produced from the Radium Solution.}

Cameron and Ramsay point out that if the emanation produces neon in the presence of water, neon should be found in the water solution of the radium salt from which the emanation is pumped off, and they state that they have observed the spectrum of neon from the gases thus obtained from the radium solution. We have made experiments to test this point. The radium salt containing over $200 \mathrm{mgrs}$. of radium was dissolved in water to which a small quantity of hydrochloric acid had been added. The emanation was allowed to collect for about five days, and was then pumped off with about 30 c.cs. of hydrogen and oxygen. These gases were treated as before, and the emanation removed by condensation. The residual gas, mostly oxygen, was tested, by the method described, for neon. We have made four experiments in all, but in no case have we found a trace of neon, although a brilliant spectrum of helium was always observed.

\section{Conclusions.}

From the rate of production of helium by radium recently measured by Sir James Dewar*, the quantity of helium produced by a known quantity of emanation can be readily estimated. In most of the experiments with water a quantity of emanation corresponding to the equilibrium amount from * Proc. Roy. Soc. A. lxxzi. p. 280 (1908).

Phil. Mag. S. 6. Vol. 16. No. 95. Nov. 1908. $3 \mathrm{H}$ 
about $150 \mathrm{mgr}$. of radium was introduced and left for three days. This should lead to the production of about $\frac{1}{10} \mathrm{c} . \mathrm{mm}$. of helium. We have seen that in these experiments we could detect with certainty a quantity of neon less than 1000 c.mm. Consequently, even supposing that the emanation does change into neon, the amount so produced cannot be more than one per cent. of the helium which is also formed. Cameron and Ramsay were apparently not aware of the delicacy of the spectroscopic detection of neon in small quantities of air. In the experiment described in their last paper, they state that they were unable to avoid a leakage of air into their apparatus, and working with $12 \cdot 6$ c.c. of mixed gases, they found after the removal of hydrogen and oxygen that the residue consisted of $\cdot 292$ c.c. of nitrogen and carbon dioxide. Assuming that this residue consisted mainly of nitrogen, it would show that there was a leakage of air of about $\cdot 36$ c.c. In our experience, the admission of such a quantity of air into the apparatus gives a brilliant spectrum of neon comparable in brightness with the companion helium spectrum. Consequently, the experiment described by. Cameron and Ramsay is quite inadequate as a proof of the production of neon from the emanation.

University of Manchester, Oct. 6, 1908.

LXVIII. On the Equilibrium of Heterogeneous Substances (1876). By the late Prof. J. Clerk Maxwele, F.R.S.

[THE paper here reprinted is the report of an Address delivered by Prof. Clerk Maxwell on May 24, 1876, at the South Kensington Conferences in connexion with the Special Loan Collection of Scientific Apparatus. It is contained (pp. 144-150) in the official volume of reports of the Conferences, which has long ago dropped out of notice. An earlier and less complete version of this summary of Prof. Willard Gibbs's developments of the doctrine of available energy, on the basis of his new concept of the chemical potentials of the constituent substances, was communicated to the Cambridge Philosophical Society on March 8, 1876, and appeared in abstract in vol. ii. of their Proceedings, pp. 427-430 ; it was reprinted in Maxwell's 'Collected Papers,' vol. ii. pp. 498-500. The fact that the energy associated with any constituent substance is proportional jointly to the mass of that constituent and to another factor representing energy per unit mass, is of course involved in the very notion of conservation of energy : but the general factorization into magnitudes (or quantities) and intensities 